> Les vésicules extracellulaires (VE) suscitent un intérêt croissant lié à leur capacité à transférer du contenu biologique entre cellules. Les $V \varepsilon$, émises dans l'espace extracellulaire, circulent via les différents fluides de l'organisme et modulent localement ou à distance les réponses des cellules avec lesquelles elles ont interagi. Des données cliniques et expérimentales étayent leur rôle dans les maladies liées au syndrome métabolique. Les $V \varepsilon$ bousculent la vision traditionnelle de la communication intercellulaire et représentent ainsi un mode de communication alternatif et versatile, qui ouvre la porte à de nouveaux concepts et opportunités tant biologiques que thérapeutiques. <

\section{Vésicules \\ extracellulaires, \\ biomarqueurs \\ et bioeffecteurs \\ du syndrome \\ métabolique}

Soazig Le Lay ${ }^{1}$, M. Carmen Martinez ${ }^{1,2}$,

Ramaroson Andriantsitohaina ${ }^{1,2}$

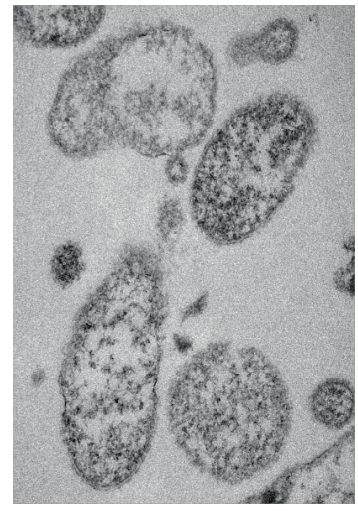

\section{Les vésicules extracellulaires}

Les vésicules extracellulaires (VE) ont longtemps été considérées comme des convoyeurs de déchets utilisés par la cellule pour se débarrasser de molécules nocives ou inutiles. Elles sont aujourd'hui reconnues comme des vecteurs de matériel biologique capables de transférer ce contenu entre cellules.

\section{Biogenèse}

Les $V \mathcal{E}$ correspondent à des nanovésicules qui sont dérivées des membranes cellulaires et sont sécrétées dans le milieu extracellulaire. Elles circulent à la faveur des nombreux fluides de l'organisme (sang, lymphe, urine, lait, etc.). Ces vésicules, hétérogènes en taille et d'origines cellulaires diverses, ont reçu de nombreuses dénominations: exosomes, microvésicules, microparticules, prostasomes, oncosomes, neurosphères, corps apoptotiques, etc. Une nomenclature a récemment été proposée par la communauté scientifique internationale distinguant essentiellement deux sous-types de VE : les microvésicules (MV) et les exosomes (Figure 1).

\section{Les exosomes}

Les exosomes (d'un diamètre compris entre 30 et $100 \mathrm{~nm}$ ) désignent des $V \varepsilon$ qui dérivent des vésicules intraluminales formées lors de la maturation des corps multivésiculaires (CMV). Elles sont sécrétées dans le milieu extracellulaire après fusion des CMV avec la membrane plasmique [1]. Leur formation implique l'assemblage de quatre complexes ESCRT (endosomal sorting complex required for transport) [1]. La biogenèse exosomale, initiée par l'accumulation de tétraspanines (CD9 et CD63) dans la membrane endosomale, est suivie du recrutement séquentiel d'ESCRT-0 et d'ESCRT-I, qui vont ségréger des cargos transmembranaires ubiquitinylés. Le recrutement du sous-complexe ESCRT-III, via ESCRT-II, induit ensuite la courbure et la fission des vésicules intraluminales (à l'origine des futurs exosomes). Une voie connexe de formation des exosomes implique les protéines syndecans et synténine, mais la participation des ESCRT dans les mécanismes régissant la sortie de ces cargos reste à déterminer. Une formation d'exosomes indépendante du complexe ESCRT a été décrite; elle fait intervenir une régulation par les tétraspanines (CD9, CD63 ou CD81) et certains lipides (des céramides) [2]. L'adressage à la membrane des exosomes formés dans les CMV et leur sécrétion engagent des protéines de trafic membranaire de la famille des petites protéines G (GTPases), telles que Rabl1, Rab35 et Rab27. Alors que Rabll et Rab35 réguleraient le trafic des vésicules endosomales précoces vers la membrane plasmique, Rab 27 interviendrait plutôt dans l'adressage à la membrane cellulaire des endosomes tardifs (lysosomes) [3].

Les exosomes participent à de nombreuses réponses physiopathologiques. Plusieurs études ont ainsi démontré leur capacité à réguler la 


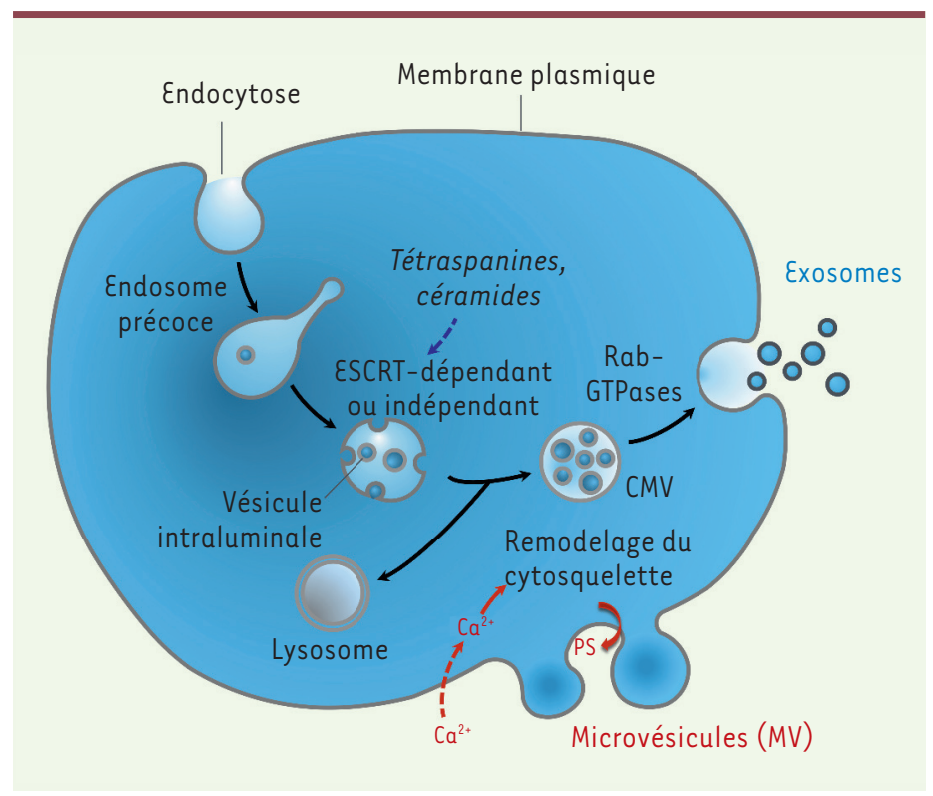

Figure 1. Voies de biogenèse des vésicules extracellulaires. Deux sous-types de vésicules extracellulaires (VE), d'origines subcellulaires différentes, peuvent être produits par les cellules eucaryotes. Les exosomes dérivent des vésicules intraluminales formées dans les corps multivésiculaires (CMV) et sont libérés dans le milieu extracellulaire à la suite de la fusion des CMV avec la membrane plasmique. Leur biogenèse est un processus contrôlé par les protéines de la voie ESCRT (endosomal sorting complexes required for transport), bien que des mécanismes indépendants aient aussi été décrits. Elle implique les protéines Rab-GTPases pour leur adressage à la surface cellulaire. Les microvésicules (MV) bourgeonnent directement à la membrane plasmique, à la suite d'un influx de calcium intracytoplasmique qui, couplé à un remodelage du cytosquelette, va conduire à l'externalisation de la phosphatidylsérine (PS) et au bourgeonnement des MV.

réponse immunitaire et anti-tumorale, notamment par leur capacité à transférer des molécules du complexe majeur d'histocompatibilité (CMH) entre cellules immunitaires [4].

\section{Les microvésicules}

Les MV, de taille comprise entre $50 \mathrm{~nm}$ et $1 \mu \mathrm{m}$ résultent d'un bourgeonnement de la membrane plasmique consécutif à un remodelage du cytosquelette lié une élévation de la concentration en calcium cytoplasmique. Cette augmentation du calcium intracellulaire stimule une machinerie enzymatique qui conduit à l'externalisation de la phosphatidylsérine (PS), un phospholipide membranaire, du feuillet interne vers le feuillet externe de la membrane plasmique [5]. La perte de l'asymétrie de la membrane qui en résulte provoque une surcharge transitoire du phospholipide aboutissant à la formation de bourgeons à partir desquels sont émises les MV. Cependant, certaines MV ne présentent pas de PS externalisée, ce qui suggère que d'autres mécanismes, encore mal caractérisés, contribueraient à la formation de ces MV. La sécrétion des MV dépend de la voie de signalisation Rho GTPase et des kinases associées à Rho. À l'image des exosomes, les MV véhiculent des messages biologiques entre de nombreux types cellulaires et sont associés au développement de diverses patho- logies. Dans des cellules spécialisées, comme les plaquettes, les MV porteuses de PS possèdent un fort potentiel coagulant. Elles ont ainsi suscitées un fort intérêt comme outils diagnostiques dans les maladies pro-thrombotiques [6].

\section{Isolement et caractérisation des sous-types de vésicules extracellulaires}

Le protocole d'isolement des VE le plus communément utilisé consiste en des centrifugations différentielles afin de concentrer les MV (entre $10000 \times g$ et $20000 \times g$ ) ou les exosomes (à $100000 \times g$ ). Cependant, cette approche, qui repose sur la différence de taille entre les deux types de vésicules, ne tient pas compte de leur origine subcellulaire et du chevauchement de leur taille (entre $50 \mathrm{~nm}$ et $1 \mu \mathrm{m}$ pour les MV et entre 30 et $100 \mathrm{~nm}$ pour les exosomes). Ainsi, la communauté scientifique internationale s'est positionnée afin de déterminer des pré-requis permettant de valider ou non l'isolement et la présence de VE dans les extraits tissulaires et les fluides corporels. Une combinaison de techniques permettant de déterminer des critères morphologiques, biophysiques et biochimiques est ainsi définie pour caractériser les sous-types de VE isolées [7]. La microscopie électronique reste la technique de référence pour déterminer la morphologie et la taille des vésicules. Des technologies récentes, fondées sur l'analyse du mouvement brownien (suivi individuel des particules - Nanosight ${ }^{\circledR}$, Malvern Panalytical) ou de la résistivité, lors du passage des vésicules au travers d'un pore (tunable resistive pulse sensing, TRPS - qNano, Izon ${ }^{\circledR}$ ), permettent désormais d'évaluer leur concentration et leur taille. Du fait de leur origine subcellulaire différente, les MV et les exosomes présentent des signatures protéiques qui leur sont spécifiques et permettent de les distinguer $[8,9]$. Des stratégies alternatives (chromatographie par exclusion de taille, immunocapture, etc.) permettent également l'isolement de $V E$, qui présenteront cependant des caractéristiques différentes de celles purifiées par centrifugation différentielle [7].

\section{Composition, adressage et interactions cellulaires des vésicules extracellulaires}

\section{Composition des vésicules extracellulaires}

Les $V \varepsilon$ sont constituées d'une bicouche lipidique délimitant un contenu varié de protéines solubles ou membranaires, de lipides, de métabolites et d'acides nucléiques. Les nombreuses analyses protéomiques, lipidomiques et génomiques (regroupées dans la base de données EVpedia [10]) ont permis de répertorier les différents cargos transportés par les vésicules. Résultat de leur processus 


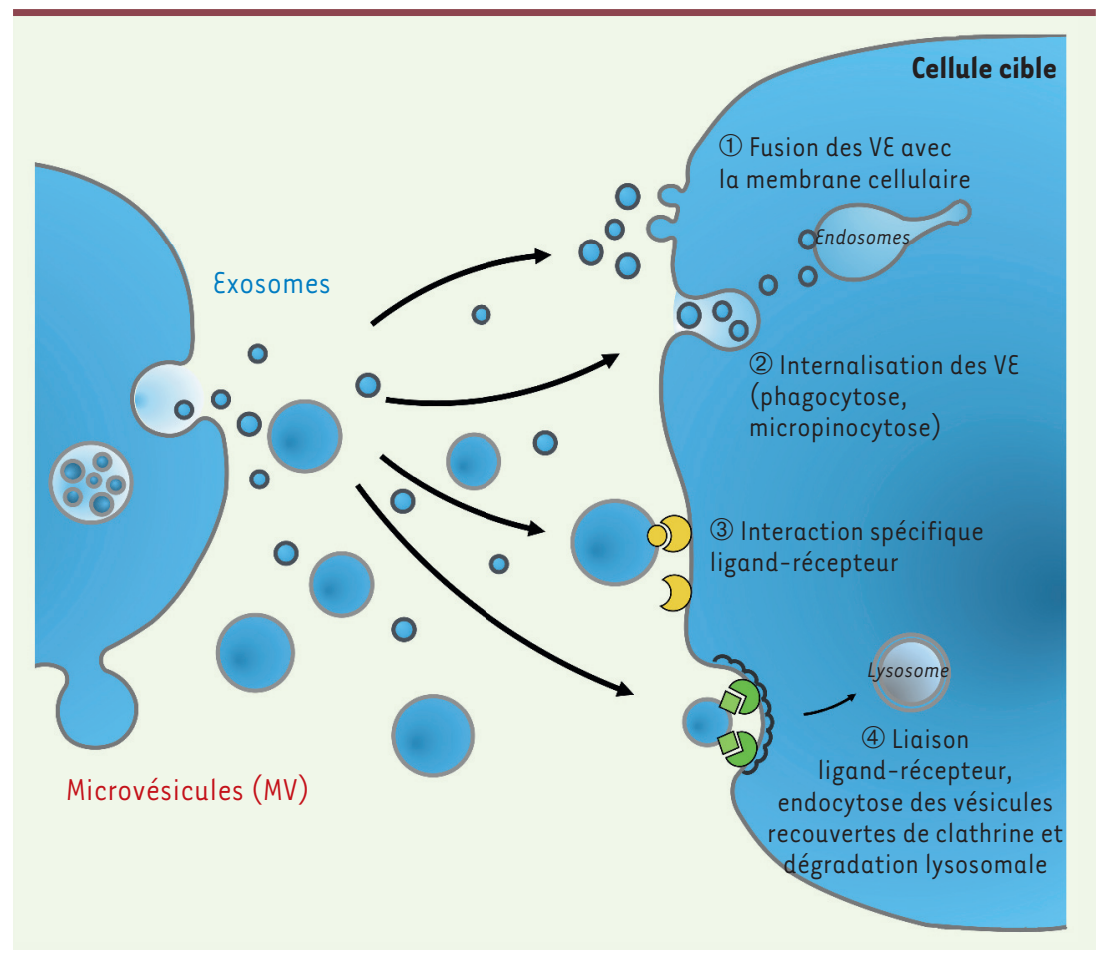

Figure 2. Mode d'action des vésicules extracellulaires sur les cellules cibles. Les vésicules extracellulaires $(V E)$ libérées dans le milieu extracellulaire circulent via les fluides de l'organisme pour interagir avec les cellules et tissus cibles. Différents mécanismes ont été décrits pour ces interactions: ( 1 ) une fusion des $V \varepsilon$ avec la membrane de la cellule cible, conduisant à la libération du contenu de la vésicule dans le cytosol (plusieurs travaux suggèrent également que la fusion se réalise au niveau des endosomes, après endocytose de la vésicule); (2) I'internalisation des VE par phagocytose ou macropinocytose; (3) I'interaction spécifique de ligands présents à la surface des $V \varepsilon$ avec des récepteurs exprimés par les cellules cibles; ou (4) I'internalisation des $V \mathcal{E}$ au niveau de puits de clathrine, à la suite d'une liaison de type ligand/récepteur, qui conduit à une dégradation des vésicules recouvertes de clathrine dans les lysosomes.

de biogenèse, les exosomes sont enrichis en protéines impliquées dans la sortie endosomale, comme les tétraspanines (Tsg101 [tumor susceptibility 101], CD9, CD63) ou Alix (ALG-2 interacting protein X). Ces marqueurs sont bien souvent utilisés pour caractériser cette population de vésicules et/ou les isoler par immuno-affinité. Cependant, différents sous-types d'exosomes peuvent co-exister, reflétant probablement des voies connexes de biogenèse [8]. Les MV présentent à leur membrane des antigènes fonctionnels et/ou des molécules d'adhérence provenant de la cellule émettrice, une propriété utilisée pour déterminer leur origine cellulaire par cytométrie en flux. Certaines familles de protéines sont toutefois enrichies dans les deux sous-types de vésicules. On retrouve ainsi des protéines du cytosquelette (actine, tubuline), du CMH, des chaperones (Hsp [heat shock protein], endoplasmine), de la matrice extracellulaire, des protéines liant les acides nucléiques (protéines ribosomales 405 et 605 , histones, facteurs d'élongation), des enzymes métaboliques (souvent mitochondriales), et des protéines impliquées dans le trafic membranaire (annexines, Rab GTPases) [9].

Différents types d'acides nucléiques (ADN, ARN, ARNm, ARN interférents ou petits $A R N$ non codants) sont également véhiculés par les $V \varepsilon$. Les mécanismes régissant la circulation de cette information génique associée aux VE restent cependant à élucider. Un adressage particulier de ces acides nucléiques vers les MV reposerait sur une séquence non codante servant de code-barres [11].

\section{Adressage et interactions cellulaires des vésicules extracellulaires}

L'interaction des MV et des exosomes avec les cellules cibles et le transfert de leur contenu modulent la réponse cellulaire. L'induction d'une activité luciférase dans des cellules dendritiques (transfectées par un système gène/rapporteur luciférase) par des exosomes chargés en luciférine illustre le transfert de protéines cytosoliques [12]. Un tel transfert peut aussi impliquer des ARNm codants ou des petits ARN interférents qui rendront silencieux des gènes de la cellule réceptrice. Les $V \varepsilon$ utilisent des mécanismes variés pour interagir avec les cellules, mécanismes qui peuvent conduire à l'activation de cascades de signalisation dans les cellules cibles. Le mode d'action des VE sur les cellules cibles dépend des protéines et des glycoprotéines qui sont présentes à la surface des $V \varepsilon$ et de celles qui sont exprimées par les cellules cibles [13] (Figure 2). Les VE peuvent ainsi se lier aux cellules via des récepteurs membranaires spécifiques. L'interaction ligand (présent sur les $V \varepsilon$ )/récepteurs peut soit stimuler des cascades de signalisation intracellulaire soit être suivi d'une internalisation des vésicules recouvertes de clathrine. La membrane des $V \varepsilon$ peut aussi fusionner directement avec la membrane de la cellule cible avec, ou non, endocytose (macropinocytose, phagocytose), et in fine libération de son contenu dans le cytosol. Elle peut également fusionner, après endocytose, avec les endosomes dans le cytoplasme.

\section{Le syndrome métabolique}

Le syndrome métabolique (SMet) désigne l'association d'un ensemble de troubles physiologiques (excès de poids, hypertension artérielle) et biochimiques (altérations glucidiques et lipidiques) qui augmentent le 


\begin{tabular}{|c|c|c|c|}
\hline & WHO & NCEP-ATPIII & IDF \\
\hline Composant prioritaire & $\begin{array}{l}\text { Insulinémie élevée } \\
\text { Deux des critères suivants: }\end{array}$ & Trois des critères suivants: & $\begin{array}{l}\text { Obésité centrale (spécifique } \\
\text { du genre et de l'ethnie) } \\
\text { Deux des critères suivants: }\end{array}$ \\
\hline Contour de taille & $\begin{array}{l}>94 \mathrm{~cm} \text { hommes } \\
>80 \mathrm{~cm} \text { femmes }\end{array}$ & $\begin{array}{l}>102 \text { hommes } \\
>88 \text { femmes }\end{array}$ & $\begin{array}{l}\geq 94 \mathrm{~cm} \text { hommes } \\
\geq 80 \mathrm{~cm} \text { femmes }\end{array}$ \\
\hline Triglycérides & $\geq 150 \mathrm{mg} / \mathrm{dL}$ & $\geq 150 \mathrm{mg} / \mathrm{dL}$ & $\geq 150 \mathrm{mg} / \mathrm{dL}$ \\
\hline HDL-cholestérol & $\begin{array}{l}<35 \mathrm{mg} / \mathrm{dL} \text { hommes } \\
<39 \mathrm{mg} / \mathrm{dL} \text { femmes }\end{array}$ & $\begin{array}{l}<40 \mathrm{mg} / \mathrm{dL} \text { hommes } \\
<50 \mathrm{mg} / \mathrm{dL} \text { femmes }\end{array}$ & $\begin{array}{l}<40 \mathrm{mg} / \mathrm{dL} \text { hommes } \\
<50 \mathrm{mg} / \mathrm{dL} \text { femmes }\end{array}$ \\
\hline Glycémie & $\geq 110 \mathrm{mg} / \mathrm{dL}$ & $\geq 110 \mathrm{mg} / \mathrm{dL}$ & $\begin{array}{l}\geq 110 \mathrm{mg} / \mathrm{dL} \\
\text { ou diabète de type } 2\end{array}$ \\
\hline Pression artérielle & $\geq 140 / 90 \mathrm{~mm} \mathrm{Hg}$ & $\geq 130 / 85 \mathrm{~mm} \mathrm{Hg}$ & $\geq 130 / 85 \mathrm{~mm} \mathrm{Hg}$ \\
\hline
\end{tabular}

Tableau I. Critères de diagnostic de syndrome métabolique (SMet) selon les organisations. WHO: World health organization ; NCEP-ATPIII: National cholesterol education program-adult treatment. Panel III ; IDF : International diabetes fundation; HDL : lipoprotéine de haute densité.

risque de diabète de type 2 , de maladies cardiovasculaires et de cancers. Malgré les efforts réalisés pour définir des critères internationaux qualifiant le SMet, des divergences quant aux valeurs de référence à retenir perdurent (Tableau I). En France, la prévalence du SMet est corrélée à celle de l'obésité, et est estimée entre 14 et $21 \%$ selon la définition de SMet retenue [14]. Cette prévalence, qui est indépendante du sexe, reste cependant inférieure à celle d'autres pays développés et est influencée par de nombreux facteurs socioéconomiques (habitudes alimentaires, sédentarité, alcoolisme, tabagisme, etc.).

\section{Les vésicules extracellulaires, biomarqueurs des différentes composantes du syndrome métabolique \\ L'ensemble des facteurs de risque qui contribuent à la genèse et au maintien du SMet influence la production de VE (Tableau II).}

\section{L'obésité}

L'obésité viscérale est considérée comme le facteur le plus délétère, car elle prédispose au développement d'une résistance à l'insuline $(\mathrm{RI})$, ce qui favorise l'accumulation de dépôts lipidiques, l'athérosclérose et l'hypertension. Chez les patients obèses, le tissu adipeux est infiltré de macrophages pro-inflammatoires qui contribuent à entretenir l'inflammation chronique de bas-grade associée à l'obésité [15]. Au cours de l'obésité, la concentration circulante de VE est augmentée [16]. Ces VE ont pour origine les plaquettes, les cellules endothéliales et les leucocytes. Le tissu adipeux sécrète également des $V \varepsilon$ en quantité importante [9]. Néanmoins, la contribution de ces VE adipocytaires dans l'augmentation des $V \varepsilon$ circulantes est à ce jour inconnue.

\section{La dyslipidémie}

Des concentrations plasmatiques élevées de cholestérol LDL (lipoprotéines de faible densité) et de triglycérides sont le reflet d'une alimentation riche en lipides et en glucides. Ces conditions induisent l'apop- tose des cellules hépatiques in vitro et des animaux nourris avec un régime riche en graisses développent des stéatoses hépatiques [17]. L'accumulation de LDL dans les monocytes-macrophages infiltrés dans la paroi vasculaire facilite leur transformation en cellules spumeuses menant à la formation de la plaque d'athérome. Les animaux hyperlipémiques sans altération de métabolisme glucidique présentent une concentration circulante de VE dérivées des monocytes élevée [18]. Des résultats similaires sont observés chez des patients hypertendus souffrant d'hyperlipémie [19].

\section{L'hypertension artérielle}

L'augmentation de la pression artérielle (PA) au cours d'un SMet reflète l'interaction complexe entre différents systèmes de régulation. La sécrétion d'angiotensine II par le tissu adipeux régule la production d'aldostérone qui joue un rôle crucial dans le contrôle de la PA [20]. L'augmentation de l'activité du système nerveux sympathique associée à l'hyperinsulinémie contribue au développement de l'hypertension.

La concentration circulante de $V \varepsilon$, principalement des MV, est augmentée dans des modèles d'animaux hypertendus [21]. En outre, plusieurs études ont montré le fort potentiel des MV d'origine endothéliale, ou plus généralement circulantes, comme marqueur pronostique/diagnostique des pathologies athérothrombotiques [6].

\section{Résistance à l'insuline et diabète de type 2}

L'activation du récepteur de l'insuline conduit à l'absorption du glucose et à l'augmentation de la synthèse 


\begin{tabular}{l} 
Obésité \\
\hline Dyslipidémie \\
\hline Hypertension artérielle \\
Résistance à l'insuline et diabète de \\
type 2
\end{tabular}

Syndrome métabolique (SMet)
[VE] circulante augmentée

Sécrétion de $V \varepsilon$ adipocytaires
$[9,16]$

$[18,19]$

$[V \varepsilon]$ circulantes augmentée chez le rat

[VE] circulantes augmentée chez l'homme

Corrélation positive entre $V \mathcal{E}$ monocytaires et sensibilité à l'insuline
[VE] plaquettaires, endothéliales et érythrocytaires augmentée Corrélation entre taux de VE exprimant le facteur tissulaire et nombre de critères du SMet

Tableau II. Utilisation des vésicules extracellulaires (VE) comme biomarqueurs potentiels pour le pronostic/diagnostic des complications métaboliques.

du glycogène et de la synthèse protéique, ce qui permet d'accroître les réserves énergétiques. L'obésité est associée au développement d'une résistance à l'insuline $(\mathrm{RI})$, ce qui se traduit par une réduction des effets métaboliques de l'hormone, à savoir un défaut d'entrée de glucose et une moindre inhibition de la lipolyse dans les tissus insulino-dépendants [22], ce qui engendre une hyperlipémie. Les lipides en excès vont alors se déposer de manière ectopique dans le foie, le muscle squelettique, le cœur ou dans les vaisseaux, conduisant à des phénomènes de lipotoxicité. Au niveau hépatique, le défaut de régulation de la glycogénolyse et la gluconéogenèse dans la RI favorise I'hyperglycémie. Au niveau de l'endothélium vasculaire, la RI réduit la production de monoxyde d'azote et augmente la vasoconstriction, avec pour résultat une hypertension. La RI est la principale cause du diabète de type 2. À long terme, la concentration élevée de glucose dans le sang affecte la fonctionnalité de certains tissus et organes (yeux, système nerveux, cœur, vaisseaux, reins, etc.).

Les concentrations circulantes de MV totales, de même que celles dérivées spécifiquement des plaquettes, des monocytes ou des cellules endothéliales, sont significativement augmentées chez les patients atteints de diabète de type 2, comparés à des individus sains [23]. Une corrélation positive entre $V \varepsilon$ monocytaires et sensibilité à l'insuline des patients diabétiques, de même qu'avec d'autres composantes du SMet, comme l'obésité et la dyslipidémie, a été récemment mise en évidence [24].

\section{Ensemble des composantes du SMet}

L'imbrication des cinq critères définissant le SMet rend difficile la détermination de la participation de chacun séparément. Cependant, il est clairement établi que les patients atteints de SMet présentent des taux de $V E$ plaquettaires, endothéliales et érythrocytaires élevés par rapport aux personnes saines $[25,26]$. Le taux de VE exprimant le facteur tissulaire (FT ou facteur III) est corrélé au nombre de critères du SMet [27] présenté par les patients, et celui de VE exprimant la cystatine C, aux complications métaboliques [28].

\section{Les vésicules extracellulaires, bioeffecteurs sur les tissus cibles}

Parallèlement à leur rôle de biomarqueurs, les VE, par leur capacité de transférer leur contenu à des cellules cibles, sont de véritables acteurs de la communication intercellulaire et pourraient jouer un rôle prépondérant dans le SMet. La réponse cellulaire induite par les $V \varepsilon$ s'avère souvent plus influencée par la qualité des $V E$, déterminée par leur origine cellulaire et leur composition, que par leur quantité (Figure 3).

\section{Effets vasculaires}

La contribution des vésicules extracellulaires plasmatiques à la dysfonction endothéliale souvent associée au SMet a été évaluée dans des expériences dans lesquelles des $V \varepsilon$ isolées à partir du plasma de patients souffrant ou non de SMet ont été injectées par voie intraveineuse à des souris. Dans ces conditions, les VE de patients présentant un SMet, comparées à des $V \varepsilon$ isolées d'individus sains, induisent chez les souris des altérations de la réponse de l'aorte à l'acétylcholine $[29,30]$. Cette réponse cellulaire induite par les $V \varepsilon$ de patients repose sur la voie de signalisation Fas/ligand de Fas [30], à l'origine de la production d'espèces réactives de l'oxygène ( $\varepsilon R 0)$ dépendant de l'activation de la sphingomyélinase neutre [29].

Par ailleurs, des concentrations élevées de glucose ou d'angiotensine-II augmentent la production de MV endothéliales présentant un potentiel pro-oxydant, pro-coagulant et pro-inflammatoire [31,32]. L'injection de ces MV à des souris reproduit les effets délétères d'une forte exposition au glucose sur les cellules endothéliales, à savoir une altération de la relaxation 


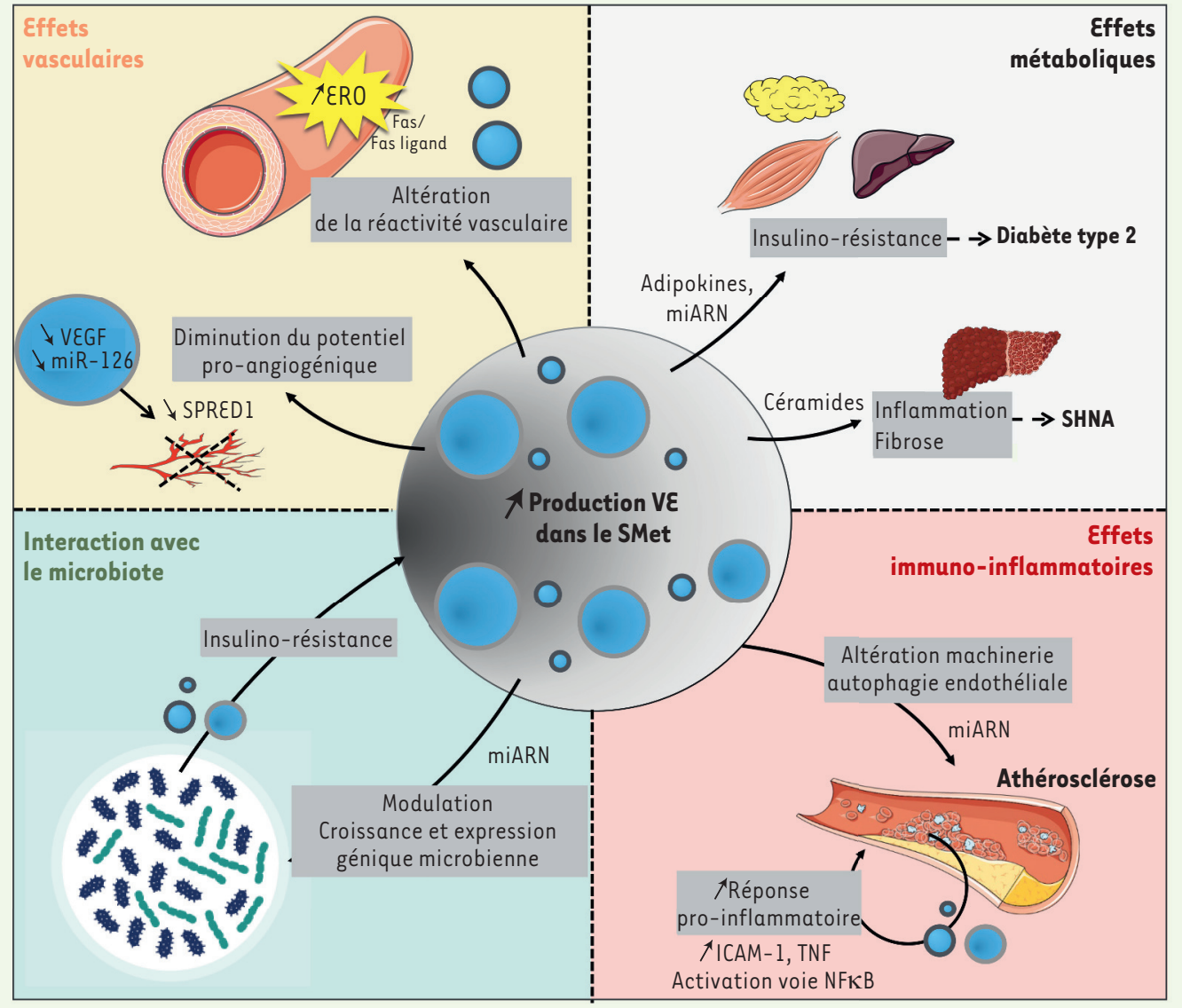

Figure 3. Les vésicules extracellulaires (VE), bioeffecteurs des complications métaboliques sur les tissus cibles. La production des VE, de diverses origines cellulaires, est souvent augmentée au cours du SMet (syndrome métabolique). Ces VE vont ainsi interagir avec différents tissus cibles et induire des dysfonctions métaboliques par des effets vasculaires, métaboliques ou immuno-inflammatoires. Ces effets peuvent être produits par différents constituants des VE (protéines, micro-ARN [miARN], lipides) qui modulent la réponse cellulaire et ainsi participent au développement des dysfonctions métaboliques associées au SMet. SHNA : stéatose hépatique non alcoolique; ERO : espèces réactives de l'oxygène ; SPREDI: sprouty-related EVHI domain-containing 1 ; VEGF : vascular endothelial growth factor; ICAM-1 : intercellular adhesion molecule- 1 ; TNF $\alpha$ : tumor necrosis factor alpha; NFKB : nuclear factor kappa B.

en réponse à l'acétylcholine et le développement de plaques athéromateuses, en induisant la production d'ERO par ces cellules [32].

L'obésité réduit considérablement le potentiel pro-angiogénique des $\checkmark \varepsilon$ dérivées des cellules mésenchymateuses adipocytaires, notamment en modulant négativement le contenu de facteurs pro-angiogéniques, comme le VEGF (vascular endothelial growth factor), ou du micro-ARN miR-126 [33, 34]. miR-126 est l'un des micro-ARN les plus abondants dans les MV dérivées des cellules endothéliales. II cible principalement, l'expression de la protéine SPREDl (sprouty-related EVHI domain containing 1) et module ainsi les voies de signalisation angiogéniques [34].

\section{Effets métaboliques}

L'injection d'exosomes dérivés d'explants de tissu adipeux de souris obèses à des souris saines est suffisante pour induire une résistance à
I'insuline associée à une activation macrophagique qui se traduit par une sécrétion accrue de cytokines proinflammatoires [35]. Outre les adipokines, qui peuvent moduler la sensibilité à l'insuline $[9,36,37]$, les exosomes dérivés du tissu adipeux transfèrent également des micro-ARN qui ciblent spécifiquement les transcrits des protéines de la signalisation insulinique et des voies de signalisation pro-inflammatoires et fibrotiques [38, 39], suggérant leur participation dans le développement du diabète de type 2 . Les souris invalidées pour le gène codant le TLR4 (Toll-like receptor 4) sont protégées contre les effets délétères des exosomes dérivés de tissu adipeux de souris obèses, ce qui montre la participation de ce récepteur de l'immunité innée dans la réponse inflammatoire que ces vésicules induisent [35]. 
Dans les conditions d'hyperlipémie caractéristiques du SMet, les acides gras saturés induisent la production de $V \varepsilon$ par les hépatocytes, les cellules musculaires et les adipocytes [9, 40, 41]. Ces VE, qui sont enrichies en céramides connus pour leur lipotoxicité, sont capables de transférer ces lipides à des cellules musculaires ou des macrophages $[40,41]$. Les VE sécrétées à la suite d'une stimulation lipidique possèdent aussi des propriétés fibrosantes et inflammatoires. De même, les exosomes adipocytaires de sujets obèses peuvent induire une réponse pro-fibrotique dans les hépatocytes [42].

L'ensemble de ces données souligne le rôle potentiel que pourraient jouer les $V \varepsilon$ dans le développement de la stéatose hépatique non alcoolique (NASH) et suggère leur utilisation à des fins pronostiques/ diagnostiques afin d'évaluer la progression de cette maladie [41].

\section{Effets sur le système immuno-inflammatoire}

L'athérosclérose, une des complications cardiovasculaires majeures du SMet, se caractérise par une inflammation chronique de l'intima artérielle marquée par l'accumulation de LDL et de cellules apoptotiques. L'oxydation des LDL et la nécrose des cellules apoptotiques vont rendre ces dépôts fortement inflammatoires et immunogènes. L'autophagie endothéliale - en nettoyant et recyclant des composants des cellules tapissant la paroi vasculaire - exerce un rôle protecteur limitant le développement des plaques athéromateuses. Des travaux récents ont mis en évidence une altération de la machinerie autophagique endothéliale par des VE dérivées de cellules musculaires lisses aortiques, capables de moduler l'expression d'acteurs clé de l'autophagie par transfert exosomal de micro-ARN [43].

Les $\vee \varepsilon$ dérivées de cellules immunitaires pourraient aussi contribuer à entretenir et amplifier la réponse inflammatoire associée à l'athérosclérose. Les exosomes, qu'ils soient dérivés de macrophages, de cellules dendritiques ou de cellules spumeuses, induisent dans les cellules endothéliales une réponse inflammatoire $[44,45]$, associée à une activation de NFKB (nuclear factor-kappa B), la production de signaux pro-inflammatoires et l'expression de molécules d'adhérence (ICAM-1, inter cellular adhesion molecule-1). Ces effets peuvent être initiés par le TNF $\alpha$ (tumor necrosis factor alpha) transporté par ces vésicules [45]. Les MV isolées de macrophages inflammatoires renferment également du TNF $\alpha$. Elles peuvent transmettre des signaux pro-apoptotiques à des cardiomyocytes en interagissant avec les cellules via leurs récepteurs du TNF $\alpha$ [46].

Les $V \varepsilon$ sont donc des bioeffecteurs qui contribuent à la propagation de signaux pro-inflammatoires. Ils favorisent l'instabilité des plaques d'athérome et conduisent à un infarctus du myocarde.

\section{Interactions hôte/microbiote via les vésicules extracellulaires}

Au cours de ces dernières années, de nombreux travaux ont révélé l'importance du microbiote intestinal dans le développement des complications métaboliques. Des dysbioses sont en effet associées à l'obésité et à la sévérité de pathologies hépatiques. Les $V \varepsilon$ représentent un relai entre l'hôte et son microbiote. Ainsi, les cellules épithéliales intestinales produisent des exosomes enrichis en certains petits ARN qui, captés par les bactéries, moduleront leur croissance et leur profil d'expression génique [47]. De même, les exosomes isolés du microbiote intestinal de souris obèses induisent une RI lorsque transférés à des souris saines
[48]. Les VE apparaissent donc comme un nouvel acteur dans la communication complexe reliant l'hôte et son microbiote.

\section{Conclusion}

Associées à plusieurs facteurs de risque cardio-métaboliques, les VE pourraient donc représenter de nouveaux biomarqueurs prédictifs de pathologies cardiovasculaires. Connaître la composition des $V \varepsilon$ et comprendre leurs possibles activités vis-à-vis de leurs cibles cellulaires pourraient permettre de concevoir des stratégies innovantes visant à moduler leur biogenèse et leurs actions. Celles-ci pourraient conduire à une personnalisation des traitements selon les sous-types de VE identifiés chez les patients, leurs compositions et leurs activités biologiques. $\diamond$

\section{REMERCIEMENTS}

Les auteurs remercient le soutien de l'Inserm, le CHU d'Angers et l'université d'Angers. Ce travail a été soutenu par la Société Francophone du Diabète et la Région Pays de Loire (Mibiogate).

\section{LIENS D'INTÉRÊT}

Les auteurs déclarent n'avoir aucun lien d'intérêt concernant les données publiées dans cet article.

\section{SUMMARY}

Extracellular vesicles as biomarkers and bioeffectors of metabolic syndrome

Extracellular vesicles ( $\left(V_{\mathrm{s}}\right)$ are emerging as a novel way of cell-to-cell communication and represent an attractive way to convey fundamental information between cells. EVs, released in the extracellular space, circulate via the various body fluids and modulate locally or remotely the cellular responses following their interaction with the target cells. Clinical and experimental data support their role as biomarkers and bioeffectors in diseases related to the metabolic syndrome. EVs thus hustle the traditional vision of intercellular communication, via an alternative and versatile mode of communication, and open the door to new concepts and opportunities from a therapeutic and biological point of view. $\diamond$

\section{RÉFÉRENCES}

1. van Niel G, D'Angelo G, Raposo G. Shedding light on the cell biology of extracellular vesicles. Nat Rev Mol Cell Biol 2018 ; 19(4) : 213-28.

2. van Niel G, Charrin S, Simoes S, et al. The tetraspanin CD63 regulates ESCRTindependent and -dependent endosomal sorting during melanogenesis. Dev Cell $2011 ; 21: 708-21$.

3. Blanc L, Vidal M. New insights into the function of Rab GTPases in the context of exosomal secretion. Small GTPases $2018 ; 9: 95-106$.

4. Thery C, Ostrowski M, Segura $\varepsilon$. Membrane vesicles as conveyors of immune responses. Nat Rev Immunol $2009 ; 9$ : 581-93. 


\section{RÉFÉRENCES}

5. Hugel B, Martinez MC, Kunzelmann C, Freyssinet JM. Membrane microparticles: two sides of the coin. Physiology (Bethesda) $2005 ; 20: 22-7$.

6. Boulanger CM, Loyer $X$, Rautou PE, Amabile N. Extracellular vesicles in coronary artery disease. Nat Rev Cardiol $2017 ; 14: 259-72$.

7. Coumans FAW, Brisson AR, Buzas $\varepsilon l$, et al. Methodological guidelines to study extracellular vesicles. Circ Res 2017 ; 120 : 1632-48.

8. Kowal J, Arras G, Colombo M, et al. Proteomic comparison defines novel markers to characterize heterogeneous populations of extracellular vesicle subtypes. Proc Natl Acad Sci U S A 2016; 113 ع968-77.

9. Durcin M, Fleury A, Taillebois $\varepsilon$, et al. Characterisation of adipocyte-derived extracellular vesicle subtypes identifies distinct protein and lipid signatures for large and small extracellular vesicles. J Extracell Vesicles $2017 ; 6: 1305677$.

10. Kim DK, Kang B, Kim OY, et al. EVpedia: an integrated database of high-throughput data for systemic analyses of extracellular vesicles. J Extracell Vesicles $2013 ; 2$.

11. Bolukbasi MF, Mizrak A, Ozdener GB, et al. miR-1289 and "Zipcode"-like sequence enrich mRNAs in microvesicles. Mol Ther Nucleic Acids $2012 ; 1$ : el0.

12. Montecalvo A, Larregina AT, Shufesky WJ, et al. Mechanism of transfer of functional microRNAs between mouse dendritic cells via exosomes. Blood $2012 ; 119$ : 756-66.

13. Mulcahy LA, Pink RC, Carter DR. Routes and mechanisms of extracellular vesicle uptake. J Extracell Vesicles $2014 ; 3$.

14. Vernay M, Salanave B, de Peretti C, et al. Metabolic syndrome and socioeconomic status in France: The French Nutrition and Health Survey (ENNS, 2006-2007). Int J Public Health 2013 ; 58 : 855-64.

15. Cancello R, Tordjman J, Poitou C, et al. Increased infiltration of macrophages in omental adipose tissue is associated with marked hepatic lesions in morbid human obesity. Diabetes 2006 ; 55 : 1554-61.

16. Stepanian A, Bourguignat L, Hennou S, et al. Microparticle increase in severe obesity: Not related to metabolic syndrome and unchanged after massive weight loss. Obesity (Silver Spring) 2013 $21(11): 2236-43$.

17. Nemes K, Aberg F. Interpreting lipoproteins in nonalcoholic fatty liver disease. Curr Opin Lipidol $2017 ; 28: 355-60$

18. Ousmaal Mel F, Martinez MC, Andriantsitohaina R, et al. Increased monocyte/neutrophil and pro-coagulant microparticle levels and overexpression of aortic endothelial caveolin-lbeta in dyslipidemic sand rat, Psammomys obesus. J Diabetes Complications $2016 ; 30: 21-9$.

19. Zu L, Ren C, Pan B, et al. Endothelial microparticles after antihypertensive and lipid-lowering therapy inhibit the adhesion of monocytes to endothelial cells. Int J Cardiol $2016 ; 202: 756-9$.

20. Yvan-Charvet L, Quignard-Boulange A. Role of adipose tissue renin-angiotensin system in metabolic and inflammatory diseases associated with obesity. Kidney international $2011 ; 79: 162-8$.

21. Lopez Andres N, Tesse A, Regnault V, et al. Increased microparticle production and impaired microvascular endothelial function in aldosterone-salt-treated rats: protective effects of polyphenols. PLoS One $2012 ; 7$ : e39235.

22. Rask-Madsen C, Kahn CR. Tissue-specific insulin signaling, metabolic syndrome, and cardiovascular disease. Arterioscler Thromb Vasc Biol $2012 ; 32$ : 2052-9.

23. Li S, Wei J, Zhang C, et al. Cell-derived microparticles in patients with type 2 diabetes mellitus: a systematic review and meta-analysis. Cell Physiol Biochem $2016 ; 39: 2439-50$.

24. Botha J, Velling Magnussen L, Nielsen MH, et al. Microvesicles correlated with components of metabolic syndrome in men with type 2 diabetes mellitus and lowered testosterone levels but were unaltered by testosterone therapy. J Diabetes Res $2017 ; 2017: 4257875$.

25. Agouni A, Lagrue-Lak-Hal AH, Ducluzeau PH, et al. Endothelial dysfunction caused by circulating microparticles from patients with metabolic syndrome. Am J Pathol $2008 ; 173: 1210-9$.

26. Helal 0 , Defoort C, Robert $S$, et al. Increased levels of microparticles originating from endothelial cells, platelets and erythrocytes in subjects with metabolic syndrome: relationship with oxidative stress. Nutr Metab Cardiovasc Dis $2011 ; 21: 665-71$.

27. Diamant M, Nieuwland R, Pablo RF, et al. Elevated numbers of tissue-factor exposing microparticles correlate with components of the metabolic syndrome in uncomplicated type 2 diabetes mellitus. Circulation $2002 ; 106: 2442-7$

28. Kranendonk ME, de Kleijn DP, Kalkhoven $\varepsilon$, et al. Extracellular vesicle markers in relation to obesity and metabolic complications in patients with manifest cardiovascular disease. Cardiovasc Diabetol 2014 ; $13: 37$

29. Safiedeen Z, Rodriguez-Gomez I, Vergori L, et al. Temporal cross talk between endoplasmic reticulum and mitochondria regulates oxidative stress and mediates microparticle-induced endothelial dysfunction. Antioxid Redox Signal 2017; 26 : 15-27.
30. Agouni A, Ducluzeau PH, Benameur T, et al. Microparticles from patients with metabolic syndrome induce vascular hypo-reactivity via Fas/Fasligand pathway in mice. PLoS One $2011 ; 6$ : e27809.

31. Burger D, Montezano AC, Nishigaki N, et al. Endothelial microparticle formation by angiotensin II is mediated via Ang II receptor type I/NADPH oxidase/ Rho kinase pathways targeted to lipid rafts. Arterioscler Thromb Vasc Biol $2011 ; 31$ : 1898-907.

32. Jansen $F$, Yang $X$, Franklin BS, et al. High glucose condition increases NADPH oxidase activity in endothelial microparticles that promote vascular inflammation. Cardiovasc Res 2013 ; 98 : 94-106

33. Togliatto $G$, Dentelli $P$, Gili $M$, et al. Obesity reduces the pro-angiogenic potential of adipose tissue stem cell-derived extracellular vesicles ( $(\mathrm{V} s)$ by impairing miR-126 content: impact on clinical applications. Int J Obes (Lond) $2016 ; 40: 102-11$

34. Jansen $F$, Yang X, Hoelscher M, et al. Endothelial microparticle-mediated transfer of MicroRNA-126 promotes vascular endothelial cell repair via SPREDI and is abrogated in glucose-damaged endothelial microparticles. Circulation $2013 ; 128: 2026-38$.

35. Deng ZB, Poliakov A, Hardy RW, et al. Adipose tissue exosome-like vesicles mediate activation of macrophage-induced insulin resistance. Diabetes $2009 ; 58: 2498-505$.

36. Kranendonk ME, Visseren FL, van Balkom BW, et al. Human adipocyte extracellular vesicles in reciprocal signaling between adipocytes and macrophages. Obesity (Silver Spring) $2014 ; 22$ : 1296-308.

37. Kranendonk ME, Visseren FL, van Herwaarden JA, et al. Effect of extracellular vesicles of human adipose tissue on insulin signaling in liver and muscle cells. Obesity (Silver Spring) $2014 ; 22: 2216-23$.

38. Thomou T, Mori MA, Dreyfuss JM, et al. Adipose-derived circulating miRNAs regulate gene expression in other tissues. Nature $2017 ; 542: 450-5$

39. Ying W, Riopel M, Bandyopadhyay G, et al. Adipose tissue macrophagederived exosomal mirnas can modulate in vivo and in vitro insulin sensitivity. Cell $2017 ; 171: 372-84$ el2.

40. Aswad H, Forterre A, Wiklander OP, et al. Exosomes participate in the alteration of muscle homeostasis during lipid-induced insulin resistance in mice. Diabetologia $2014 ; 57$ : 2155-64

41. Hirsova P, Ibrahim SH, Krishnan A, et al. Lipid-induced signaling causes release of inflammatory extracellular vesicles from hepatocytes. Gastroenterology 2016; $150: 956-67$

42. Koeck $\varepsilon S$, lordanskaia T, Sevilla S, et al. Adipocyte exosomes induce transforming growth factor beta pathway dysregulation in hepatocytes: a novel paradigm for obesity-related liver disease. J Surg Res 2014 ; 192 : 268-75

43. Li L, Wang Z, Hu X, et al. Human aortic smooth muscle cell-derived exosomal miR-221/222 inhibits autophagy via a PTEN/Akt signaling pathway in human umbilical vein endothelial cells. Biochem Biophys Res Commun 2016 ; 479 : 343-50.

44. Osada-Oka M, Shiota M, Izumi Y, et al. Macrophage-derived exosomes induce inflammatory factors in endothelial cells under hypertensive conditions. Hypertens Res $2017 ; 40: 353-60$.

45. Gao W, Liu H, Yuan J, et al. Exosomes derived from mature dendritic cells increase endothelial inflammation and atherosclerosis via membrane TNFalpha mediated NF-kappaB pathway. J Cell Mol Med $2016 ; 20: 2318-27$.

46. Milbank $\varepsilon$, Soleti $R$, Martinez $\varepsilon$, et al. Microparticles from apoptotic RAW 264.7 macrophage cells carry tumour necrosis factor-alpha functionally active on cardiomyocytes from adult mice. J Extracell Vesicles 2015 ; 4 : 28621.

47. Liu S, da Cunha AP, Rezende RM, et al. The host shapes the gut microbiota via fecal microRNA. Cell Host Microbe $2016 ; 19: 32-43$.

48. Choi Y, Kwon Y, Kim DK, et al. Gut microbe-derived extracellular vesicles induce insulin resistance, thereby impairing glucose metabolism in skeletal muscle. Sci Rep 2015 ; 5 : 15878

\section{TIRÉS À PART}

R. Andriantsitohaina

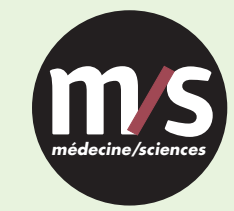

Tarifs d'abonnement m/s - 2018

Abonnez-vous

à médecine/sciences
> Grâce à $m / s$, vivez en direct les progrès des sciences biologiques et médicales

Bulletin d'abonnement page 1010 dans ce numéro de $\mathrm{m} / \mathrm{s}$

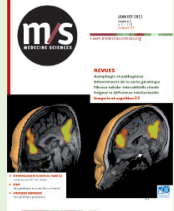

